\title{
A Male-ABCD Algorithm for Hepatocellular Carcinoma Risk Prediction in HBsAg Carriers
}

\section{Yuting Wang}

State Key Lab of Molecular Oncology, National Cancer Center/Cancer Hospital, Chinese Academy of Medical Sciences and Peking Union Medical College

\section{Minjie Wang}

Department of Clinical Laboratory, National Cancer Center/Cancer Hospital, Chinese Academy of Medical Sciences and Peking Union Medical College

$\mathrm{He} \mathrm{Li}$

Office of Cancer Screening, National Cancer Center/Cancer Hospital, Chinese Academy of Medical Sciences and Peking Union Medical College

\section{Kun Chen}

State Key Lab of Molecular Oncology, National Cancer Center/Cancer Hospital, Chinese Academy of Medical Sciences and Peking Union Medical College

\section{Hongmei Zeng}

National Central Cancer Registry, National Cancer Center/Cancer Hospital, Chinese Academy of Medical Sciences and Peking Union Medical College

\section{Xinyu Bi}

Department of Hepatobiliary Surgery, National Cancer Center/Cancer Hospital, Chinese Academy of Medical Sciences and Peking Union Medical College

\section{Zheng Zhu}

Department of Diagnostic Radiology, National Cancer Center/Cancer Hospital, Chinese Academy of Medical Sciences and Peking Union Medical College

\section{Yuchen Jiao}

State Key Lab of Molecular Oncology, National Cancer Center/Cancer Hospital, Chinese Academy of Medical Sciences and Peking Union Medical College

\section{Yong Wang}

Department of Ultrasonography, National Cancer Center/Cancer Hospital, Chinese Academy of Medical Sciences and Peking Union Medical College

Jian Zhu

Qidong Liver Cancer Institute \&amp; Qidong People's Hospital

\section{Hui Zhao}

Lingbi Center for Disease Control and Prevention

\section{Xiang Liu}

Mengcheng Center for Disease Control and Prevention

Chunyun Dai

Sheyang Center for Disease Control and Prevention

\section{Chunsun Fan}

Qidong Liver Cancer Institute \&amp; Qidong People's Hospital

Can Zhao

Shenqiu County Center for Disease Control and Prevention

\section{Deyin Guo}

Dancheng Center for Disease Control and Prevention

\section{Hong Zhao}

Department of Hepatobiliary Surgery, National Cancer Center/Cancer Hospital, Chinese Academy of Medical Sciences and Peking Union Medical College

\section{Sizhen Wang}


Genetron Health هBeijing) Co. Ltd.

Jianguo Zhou

Department of Hepatobiliary Surgery, National Cancer Center/Cancer Hospital, Chinese Academy of Medical Sciences and Peking Union Medical College

\section{Dongmei Wang}

State Key Lab of Molecular Oncology, National Cancer Center/Cancer Hospital, Chinese Academy of Medical Sciences and Peking Union Medical College

\section{Zhiyuan Wu}

State Key Lab of Molecular Oncology, National Cancer Center/Cancer Hospital, Chinese Academy of Medical Sciences and Peking Union Medical College

\section{Xinming Zhao}

Department of Diagnostic Radiology, National Cancer Center/Cancer Hospital, Chinese Academy of Medical Sciences and Peking Union Medical College

\section{Wei Cui}

Department of Clinical Laboratory, National Cancer Center/Cancer Hospital, Chinese Academy of Medical Sciences and Peking Union Medical College

\section{Xuehong Zhang}

Channing Division of Network Medicine, Department of Medicine, Brigham and Women's Hospital and Harvard Medical School

\section{Jianqiang Cai}

Department of Hepatobiliary Surgery, National Cancer Center/Cancer Hospital, Chinese Academy of Medical Sciences and Peking Union Medical College

\section{Wanqing Chen}

Office of Cancer Screening, National Cancer Center/Cancer Hospital, Chinese Academy of Medical Sciences and Peking Union Medical College

\section{Chunfeng Qu ( $\sim$ quchf@cicams.ac.cn)}

Cancer Hospital, Chinese Academy of Medical Sciences and Peking Union Medical College https://orcid.org/0000-0001-9973-0887

\section{Research Article}

Keywords: Hepatocellular Carcinoma, Hepatitis B virus, Asymptotic HBsAg Carriers, Risk prediction model, Risk assessment, Risk stratification, Screening, Laboratory Tests, des-y-carboxy-prothrombin, a-fetoprotein

Posted Date: February 9th, 2021

DOI: https://doi.org/10.21203/rs.3.rs-159806/v1

License: (c) (i) This work is licensed under a Creative Commons Attribution 4.0 International License. Read Full License 


\section{Abstract}

BACKGROUND: Hepatocellular carcinoma (HCC) development among hepatitis B surface antigen (HBsAg) carriers shows gender disparity, influenced by underlying liver diseases that display variations in laboratory tests. We aimed to construct a risk-stratified HCC prediction model for HBsAg-positive male adults.

METHODS: HBsAg-positive, 35-69 years males ( $N=6$ 153) were recruited from a multi-center population-based liver cancer screening study. Randomly, three centers were set as training, the other three centers as validation. Within 2 years since initiation, we administrated at least two rounds of HCC screening using B-ultrasonography and a-fetoprotein (AFP). We used logistic regression models to determine potential risk factors, built and examined the operating characteristics of a point-based algorithm for HCC risk prediction.

RESULTS: With 2 years of follow-up, 302 HCC cases were diagnosed. A male-ABCD algorithm was constructed including participant's Age, $\underline{B}$ lood levels of GGT (y-glutamyl-transpeptidase), Counts of platelets, white cells, $\underline{C}$ oncentration of $\underline{D} C P$ (des-y-carboxy-prothrombin) and AFP, with scores ranging from 0 to 18.3. The area under receiver operating characteristic was $0.91(0.89-0.93)$, larger than existing models. At 1.5 points of risk-score, $26.10 \%$ of the participants in training, $14.94 \%$ in validation were recognized at-low-risk, with sensitivity of identifying HCC remained $100 \%$. At 2.5 points, $46.51 \%$ of the participants in training, 33.68\% in validation were recognized at-low-risk with $99.06 \%$ and $97.78 \%$ of sensitivity. At 4.5 points, only $20.86 \%$ of training, $23.73 \%$ of validation were recognized at-high-risk, with positive prediction value of $22.85 \%$ and $12.35 \%$ respectively.

DISCUSSION: Male-ABCD algorithm identified individual's risk for HCC occurrence within short-term for their HCC precision surveillance.

\section{Introduction}

Cirrhotic patients are at particularly high-risk of hepatocellular carcinomas (HCC), a leading cause of cancer-related death worldwide[1]. HCC surveillance every six months using B-ultrasonography (US) with or without determining serum a-fetoprotein (AFP), US/AFP is strongly recommended by professional societies including Asian-Pacific Association for the Study of the Liver (APASL)[2], American Association for the Study of Liver Disease (AASLD), European Association for the Study of the Liver (EASL). Hepatitis B virus (HBV) causes HCC in absence of cirrhosis[1, 3], AASLD updates the recommendations of HCC surveillance to all HBV chronically infected adults. In China, chronic HBV infection remains the leading risk factor of HCC[1]. Results of a HCC-screening demonstration program using US/AFP biannually to HBsAgpositive adults in some rural communities of China showed no reduction in liver cancer mortality within first 4 years of follow-up[4]. Antiviral therapy rarely cures HBV infection and needs life-long medications, considerable carriers with seropositive for hepatitis B surface antigen (HBsAg) did not receive the therapy[1,5]. Worldwide, there were 257-291 million HBV chronically infected adults in 2015[5]. Tailoring to individual HCC risk is required for precision surveillance, that the limited HCC-screening resources could be allocated to high-risk individuals who might benefit most from early intervention or intensive surveillance[6].

In HBV chronically infected Asians, HCC incidence is different with variated risk factors and underlying liver diseases[1], the incidence rate was 3.2 per 100 person-years in patients with cirrhosis, and 0.4 without cirrhosis[7, 8]. To predict long-term HCC occurrence in Asians, several HCC-risk models were constructed including REACH-B[9], CU-HCC[10], GAG-HCC[11], AGED[12], that weighted significantly on HBV replication status, i.e. HBV-DNA, HBV e antigen (HBeAg). With the usage of nucleos(t)ide analogs that inhibit HBV replication, PAGE-B for Caucasian[13], mPAGE-B for Asians[14] were constructed, which employed patient's age, gender, baseline platelet (PLT) counts, and serum albumin levels, that reflect the underlying liver diseases. EASL recommends HCC surveillance to non-cirrhotic HBV patients according to PAGE-B classes. Nevertheless, these models were mostly tested in patients with chronic hepatitis B by 2019[15], the evidences of current modification in surveillance strategy based on risk stratification models are insufficient for high-grade recommendation. None of these models was tested in Chinese HBsAg-carriers from rural community where liver cancer incidence was $20.0 / 10^{5}$, higher than the urban $\left(16.1 / 10^{5}\right)[16]$.

Previous studies conducted in rural community showed that $<40 \%$ of the HBsAg-positive adults complied to the biannually repeat US/AFP tests after initial 2-3 years for HCC-screening[4, 17]. HCC development depends on presence of premalignant cells and significantly influenced by the underlying severity and activity of liver diseases $[1,18]$. Molecular and pathology analyses displayed that preneoplastic lesions harbored genetic alteration and abnormal expression of cellular proteins. For routine surveillance in community population, the risk prediction models of short-term HCC occurrence will avoid ineffective and wasteful distribution of demanding screening efforts to those at relative low-risk individuals[6]. HBV-associated HCC developed more frequently in men than in women, with a female/male ratio of 1:4 7, due to the different effect of androgen and estrogen on HBV pathogenesis and carcinogenesis[3, 19, 20]. In addition, healthy women have higher platelet and white blood cell counts than men[21]. Serum level of alanine aminotransferase (ALT) in healthy Chinese men was higher than women[22]. The currently constructed HCC prediction models used the same variation and weigh in both gender of these laboratory tests, which reflect liver diseases at different severity and activity. In this study, based on a large population-level screening program in rural 
China, we aimed to build up a risk-stratified HCC prediction model for HBsAg-positive males using measurable tests which are currently performed in clinical laboratories.

\section{Materials And Methods}

\section{Participants and study design}

The study participants were derived from the cohort of Community-based Cohort study on Population with high risk of Liver Cancer (Chinese Clinical Registry, ChiCTR-EOC-17012853), which was described previously[23]. At baseline, the 35-69 years old individuals who were immunochromatographic strip-tested positive for HBsAg were included in the cohort. Face to face interviews were conducted, which collected the sociodemographic information, body weight and height, lifestyle (smoking, drinking, eating habits, etc.), and family history of liver cancer among first-degree relatives. HCC screening by US/AFP was provided annually since 2017. The study protocol (NCC201709011) was approved by Ethics Committee of National Cancer Center/ Cancer Hospital, Chinese Academy of Medical Sciences (NCC/CH-CAMS) in Beijing. Each participant provided written informed consent before undertaking any study-related procedures in accordance with Good Clinical Practice and principles of the Declaration of Helsinki.

In the present study, we recruited the HBsAg-positive males, from six HCC-screening centers of three Provinces who meet the inclusion criteria: 1) males at 35-69 years old; 2) HBsAg-positivity; 3) no previously diagnosed HCC or other malignant diseases; 4) no other diseases that restricted taking the examinations of dynamic Computed Tomography (CT) or Magnetic Resonance Imaging (MRI). Three screening centers (Lingbi, Mengcheng, and Sheyang) were randomly assigned as training cohort, the other three centers (Shenqiu, Dancheng and Qidong) as validation cohort. (Fig. 1).

\section{Laboratory tests}

At baseline, for each participant, we measured PLT and white blood cell (WBC) counts; blood levels of ALT, AST, Y-glutamyl transpeptidase (GGT), ALB; and HCC biomarkers of AFP and des-y-carboxy-prothrombin (DCP). For the participants in training cohort, we also detected their blood concentrations of HBV-DNA, HBsAg and HBeAg. PLT, WBC, ALT, AST, ALB and AFP were measured immediately after blood drawn in each screening center independently using unified reagents. Using commercialized Abbott reagents, blood levels of GGT, concentrations of HBV-DNA, HBsAg, HBeAg, DCP were determined, and AFP was double-checked in the central laboratory of NCC/CH-CAMS using the plasma that were snap frozen in $-80^{\circ} \mathrm{C}$ for 1-2 months. HBV-DNA concentration was determined by quantitative Real-Time PCR using the reagents from Kehua Biotechnology in Roche LightCycler 480 II.

\section{Screening, follow-ups and HCC diagnosis}

Every year, the individuals were offered US/AFP examination and defined as US/AFP-positive, US/AFP-suspected, or US/AFP-negative. "US/AFP-positive" individuals had any one of the following: 1) serum AFP levels of $>400 \mathrm{ng} / \mathrm{mL}$ regardless of US-detected nodule; 2) USdetected nodule of $\geq 2 \mathrm{~cm}$ in size regardless of serum AFP concentration; and 3 ) US detected nodule of $\geq 1 \mathrm{~cm}$ in size with serum AFP $\geq$ $200 \mathrm{ng} / \mathrm{mL}$. The "US/AFP-suspected" individuals had either of the following: 1) serum AFP levels of $\geq 20 \mathrm{ng} / \mathrm{mL}$ regardless of US detected liver nodule; and 2) US-detected nodule of $\geq 1 \mathrm{~cm}$ in size. The "US/AFP-negative" individuals were defined as having serum AFP levels of < $20 \mathrm{ng} / \mathrm{mL}$ without an US-detected liver nodule. The individuals with "US/AFP-positive" and "US/AFP-suspected" were referred to specialists for HCC confirmation diagnosis. All diagnosed HCC cases were ascertained by dynamic CT or MRI. CT/MRI images were independently evaluated by two radiologists from the NCC/CH-CAMS. When diagnosis was unconfirmed, the participants were offered and volunteered for US/AFP examination 3-6 months later. We also offered the examination to $20 \%-30 \%$ of US/AFP-negatives 6 months later based on a randomized number after grouping $35-44,45-54,55-64$, and $\geq 65$ years old.

At last follow-up in 2019 , all the individuals with risk-score of $>4.5$ points, $25 \%$ of those with $1.6-2.5$ points, and $15 \%$ of those with $\leq 1.5$ points were offered and volunteered a dynamic CT/MRI examination, and all others were offered US/AFP examination. We further linked our data with local population-based cancer registries and bureaus of vital statistics of each center and confirmed liver cancer outcome (ICD-10 code C22.0 or C22.9) by Dec. 31, 2019.

\section{Model Development and Validation}

All variables at baseline were first evaluated with unconditional univariate logistic regression analysis. The variables with $P<0.1$ in univariate analysis were further assessed by stepwise multivariable logistic regression. Variables with $P<0.05$ in multivariable logistic regression were subsequently included. The discrimination was evaluated by the area under the receiver operating characteristic curve (AUROC) and its $95 \%$ confidence interval $(95 \% \mathrm{Cl})$. Calibration was assessed by the Hosmer-Lemeshow test. 
Then we created a point-based prediction rule based on coefficient-based scoring method. The coefficient of each variable was divided by the smallest coefficient in the model and rounding to the nearest 0.1 . In addition to overall performance, discrimination and calibration, the performance of the prediction rule was assessed according to its accuracy, including sensitivity, positive predictive value (PPV), negative predictive value (NPV), and the proportion of individuals of each risk-group at different cutoff of risk score.

We validated the model internally using bootstrap procedure in the training cohort by sampling with the replacement for 1000 iterations. The model was further externally validated in the independent validation cohort. With the same cutoff values defined in the training cohort, we measured the sensitivity, PPV, NPV, and the model predicted proportion of individuals in each risk group.

The analyses were performed using R software version 3.6.0 (R Foundation for Statistical Computing, Vienna, Austria).

\section{Results}

\section{Participant Demographic}

From six HCC-screening centers of three Provinces, a total of $6876 \mathrm{HBsAg-positive} \mathrm{(confirmed} \mathrm{with} \mathrm{Abbott} \mathrm{reagents)} \mathrm{males} \mathrm{were} \mathrm{recruited} \mathrm{at}$ baseline in 2017. We excluded 723 male participants, of them 87 were diagnosed with HCC before baseline, 70 were diagnosed with the other cancers, and 70 died from the causes other than HCC during the study period, and 496 were lost follow-up. At the last follow-up in Oct. 2019, a total of 6153 eligible HBsAg-positive males were included in our analyses. The training cohorts recruited $3629 \mathrm{HBsAg}$-positive males with 212 HCCs occurring. Validation cohort recruited 2524 HBsAg-positive males with 90 HCCs occurring (Fig. 1). The distributions in participants' age and the other factors variated significantly at baseline among cohorts (Table 1).

\section{Male-ABCD algorithm development and performance}

All parameters in the model development and validation were based on the baseline tests. In univariate analysis, the following variables showed significance of $P<0.1$ and they were used for further model selection (age, AFP, DCP, GGT, PLT, WBC, ALB, ALT, AST, HBV-DNA) (Supplementary table 1). In stepwise multivariate logistic regression, only age and five laboratory variables entered into the last model (Table 2). Based on these six variables, a coefficient-based scoring method was employed to develop the score-based prediction rule, which was named Male-ABCD algorithm, that represents participant's $\underline{A}$ ge, $\underline{B}$ lood levels of GGT, $\underline{C}$ ounts of PLT, WBC, and $\underline{C}$ oncentration of $\underline{D} C P, A F P$. Fig. $2 a$ shows the sensitivity and specificity of different combinations. AUROC of coefficient-based Male $A B C D$ was $0.93(0.92-0.95)$, that was higher than the AFP and DCP combination (AUROC=0.84), or the combination with age in addition (AUROC=0.88). The $\chi 2$ of the HosmerLemeshow tests was $3.77(P=0.88)$. The algorithm was converted into a point-based prediction rule, by dividing the coefficient of each variable by the smallest coefficient in the model $(0.85)$ and rounding to the nearest 0.1 (Table 2).

\section{Point-based HCC prediction and stratification by Male-ABCD}

For each given participant, the risk-score was calculated based on the Male-ABCD algorithm. At different cutoff of risk-score, $M a l e-A B C D$ grouped the HBsAg-positive males into variated proportion of HCC at-high-risk or at-low-risk. Table 3shows the sensitivity of identifying HCC within 2 years when cutoff of risk-score was set at different points, and PPV among the group at-high-risk, NPV at-low-risk. The performance of the Male-ABCD in training cohort was compared with previously developed risk-stratification models of AGED[12], REACH-B[9], PAGE-B[13], mPAGE-B[14] for general hepatitis B patients, and THRI[24] for cirrhotic patients and GALAD[25] for HCC diagnosis among high-risk population. Male-ABCD showed better AUROC over AGED, REACH-B, PAGE-B, mPAGE-B, and THRI (Fig. 2b).

\section{Validation of Male-ABCDalgorithm}

The Male-ABCD was validated in external cohort of $2524 \mathrm{HBsAg-positive} \mathrm{males} \mathrm{(Table} \mathrm{3).} \mathrm{At} 1.5$ points of risk-score, prediction reached 100\% of sensitivity both in training and validation. At 2.5 points, $46.51 \%$ of the participants in training, $33.68 \%$ in validation were recognized at-lowrisk with $99.06 \%$ and $97.78 \%$ of HCC prediction sensitivity respectively. The NPV among the population at-low-risk was $99.88 \%$ and $99.76 \%$. At 4.5 points, $20.86 \%$ of the participants in training, $23.73 \%$ in validation were recognized at-high-risk. The PPV among the population at-highrisk reached $22.85 \%$ in training and $12.35 \%$ in validation (Table 3 ).

\section{Comparison of Male-ABCD with AGED, REACH-B, PAGE-B, mPAGE-B, THRI and GALAD}

For HCC prediction and stratification, Male-ABCD was compared with previously constructed models among the total of $6153 \mathrm{HBs} A g-p o s i t i v e$ males (Table 4). Male-ABCD generated the largest AUROC in comparison with the models of AGED[12], REACH-B[9], PAGE-B[13] and mPAGE$\mathrm{B}[14]$ that were constructed based on hepatitis B patients, with THRI[24] that was based on cirrhotic patients, and with GALAD[25] that was used for HCC early diagnosis among high-risk population. For risk-stratification, Male-ABCD predicted $21.52 \%$ of total HBsAg-positive males into HCC at-low-risk with $100 \%$ of sensitivity at 1.5 points of risk-score. With $100 \%$ of prediction sensitivity, REACH-B recognized $4.27 \%$ and 
PAGE-B predicted $3.17 \%$ of total as HCC at-low-risk. At 2.5 points of risk-score, Male-ABCD predicted $41.25 \%$ of total HBsAg-positive males into HCC at-low-risk with $98.68 \%$ of sensitivity, and the NPV reached $99.84 \%$. The missed four HCCs were detected $6.1,9.9,9.9,19.9$ months later since the baseline tests respectively. While, AGED predicted 6.37\%, and mPAGE-B predicted $9.25 \%$ of total into HCC at-low-risk with $99.06 \%$ and $99.01 \%$ of sensitivity respectively, $99.13 \%$ and $99.47 \%$ of NPV respectively. With risk-score of $2.5-4.5$ points based on $\mathbf{M a l e}-\mathbf{A B C D}$, $25.50 \%(13 / 51)$ HCCs were detected between 12-24 months.

\section{Discussion}

The current study was based on a large population-level screening program, recruited 6153 HBsAg-positive male adults from six HCCscreening centers of three Provinces in rural China. In this perspective, multicenter study, we developed and validated an HCC risk-stratified algorithm named Male-ABCD, which represents participant's Age, Blood levels of GGT, Counts of PLT, WBC, and Concentration of DCP, AFP. Based on this algorithm, for a given HBsAg-positive male, his HCC risk within 2 years was recognized at-low-risk when the risk-score was $\leq$ 2.5 points, particularly $\leq 1.5$ points. However, his HCC risk within 2 years was very high when the risk-score reached $>4.5$ points. For the population of HBsAg-positive male adults with the point-based prediction, 1324 of total 6153 HBsAg-positives (21.52\%) were grouped into HCC at-low-risk when risk-score was $\leq 1.5$ points, none HCC occurred within 2 years. At 2.5 points of cutoff, 2538 of 6153 (41.25\%) HBsAgpositives were recognized HCC at-low-risk, only four HCCs occurred within 2 years. The sensitivities were $99.06 \%$ in training, $97.78 \%$ in validation with $99.88 \%$ and $99.76 \%$ of NPV respectively. At 4.5 points of cutoff, $22.04 \%$ (1 356/6 153) of the HBsAg-positives were recognized as HCC at-high-risk within 2 years with 18.22\% of PPV. After head-to-head comparison with previously developed models of AGED[12], REACH-B[9], PAGE-B[13], mPAGE-B[14] for general hepatitis B patients, THRI[24] for cirrhotic patients, and GALAD[25] for HCC diagnosis among high-risk population, Male-ABCD generated the largest AUROC. Our current study indicated that HBsAg-positive male adults could be identified at different HCC risk based on Male-ABCD algorithm. The tests employed in are measurable and currently performed in clinical laboratories. As barriers in the community to screen all HBsAg carriers biannually, Male-ABCD algorithm provided an applicable model for identifying the higher risk group who truly need intense screening intervals.

Several studies in clinic-based patients indicated that the combination of AFP/DCP, which are employed in the GALAD for early HCC diagnosis[25], discriminates early HCC from benign chronic liver diseases[1, 26, 27]. AFP has been widely used for HCC surveillance[28], DCP elevation was observed several months before HCC occurred[23, 26]. When cutoff values were fixed at AFP $=20 \mathrm{ng} / \mathrm{ml}, \mathrm{DCP}=40 \mathrm{mAU} / \mathrm{ml}$, the combination well discriminate HCC from benign liver diseases 12 months before the clinical HCC diagnosis based on patients of two genders[21]. Among the HBsAg-positive males, we observed that HCC risk increased when DCP $\geq 20 \mathrm{mAU} / \mathrm{ml}$ and AFP $\geq 7 \mathrm{ng} / \mathrm{ml}$. We didn't include AFP-L3 because our previous results indicated that AFP-L3 addition decreased the sensitivity of HCC discrimination from cirrhosis[27]. Increase of serum GGT is related to liver inflammation and was also recognized as an independent HCC risk factor[29, 30]. We previously reported that GGT with some clinical factors predicted 3-year HCC occurrence among HBsAg-positive individuals[29]. Our current study showed that GGT at $20 \sim 44 \mathrm{U} / \mathrm{L}$ and $\geq 80 \mathrm{U} / \mathrm{L}$ showed similar impact, but higher impact at $45 \sim 79 \mathrm{U} / \mathrm{L}$ on $\mathrm{HCC}$ risk. PLT is a critical indicator of cirrhosis, that are at particularly high-risk of $\mathrm{HCC}[24,25,28]$. Our study showed that adding these laboratory tests that reflect the underlying severity of liver diseases to Age/AFP/DCP, the algorithm significantly improved the sensitivity and risk-stratification among the HBsAg-positive male adults.

The current Male-ABCD was constructed base on the HBsAg-positive male adults from community population instead of the patients from hospitals. HBV have long-term effects on HCC, that higher levels of serum HBV-DNA and HBsAg showed increased HCC risk in the models of REACH-B, GAG-HCC $[9,11,29]$. We failed to observe their impact on HCC occurrence in short-term. It have been documented that normal values of ALT and PLT in healthy males are different from healthy females[21, 22]. We failed to observe the significant effect of elevated blood levels of AST and ALT in REACH-B[9], and decreased blood albumin levels in CU-HCC[10], mPAGE[14] as the short-term HCC risk factor for the HBsAg-positive males. For 2-year HCC occurrence prediction, the head-to-head comparison with previously constructed REACH-B[9], AGED[12], PAGE-B[13], mPAGE-B[14], THRI[24] and GALAD[25], Male-ABCD showed better performance for HBsAg-positive males in China rural community.

With the risk-assessment using Male-ABCD, the HBsAg-positive males were grouped into at-high-risk, or at-low-risk. This discrimination would be helpful to take different strategy to monitor their HCC. Based on Male-ABCD, none HCC occurrence among the 1324 of total 6153

(21.52\%) HBsAg-positive males with risk-score of $\leq 1.5$ points. Instead of the biannually examination, the annually or longer interval followup would reduce the total US-examination numbers to avoid over-screening, and relieve the participant anxiety. However, 22\% (1 356/6 153) of the HBsAg-positive males were recognized with the risk-score of $>4.5$ points and the PPV reached to $18.22 \%$. These population of HCC athigh-risk should be strictly followed the recommendation by professional societies, that is US/AFP examination every six months[2].

\section{Limitation}


The Male-ABCD is constructed based on HBsAg-positive males. The applicability for HBsAg-positive females may not be suitable, and needs to be evaluated in the future. No significant impact of higher levels of HBV-DNA on HCC occurrence in short-term was observed, that potentially provide a window time for the standardized antiviral therapy to reduce HCC risk[9-12] as around 80\% of the participants in our study received no and/or not-standardized antiviral therapy. With the reduced cost and more effective antiviral medicines used, the applicability of Male-ABCD needs to be further evaluated.

Different from REACH-B[9], AGED[12], PAGE-B[13], mPAGE-B[14] and THRI[24], Male-ABCD was unable to class the HBsAg-positive males into $\mathrm{HCC}$ at-highest and at-middle-high risk respectively. However, when risk-score was $>4.5$ points based on Male-ABCD, our model recognized around $22 \%$ of HBsAg-positive males into HCC at-high-risk with $18.22 \%$ of PPV.

\section{Conclusions}

The Male-ABCD algorithm could identify individual HCC risk within 2 years among HBsAg-positive males since initial laboratory tests. All tests are currently performed in clinics with an immediate clinical applicability for their HCC precision surveillance.

\section{Declarations}

Author contributions: C.Q., and Wanqing Chen designed research, supervised the study. C. Q, Yuting Wang, Minjie Wang, K.C performed the laboratory examinations. C.Q., Hongmei Zeng, Wanqing Chen, X. B., supervised the recruitment of participants and follow-up. Z.Z, Jian Zhu, Hui Zhao, X.L., C. D., C. F., C. Z., D, G., Hong Zhao, Yong Wang, Jianguo Zhou, D. W., Z, W., X. Z., J. C., performed cohort management, sample collection and clinical evaluation of the potential HCC patients. C.Q., Yuting Wang, Hongmei Zeng, Wanqing Chen, H. L., set up the model to predict HCC. X.Z. instructed the model development and statistics. Y.J., S. W. participated in statistics. C. Q., Yuting Wang, Minjie Wang, K. C., Wei Cui supervised the laboratory examination. C.Q., Wanqing Chen, Yuting Wang, Hongmei Zeng, H. L. drafted and revised the manuscript. X.Z. revised the manuscript. C.Q. and Wanqing Chen finalized the manuscript.

Funding: This study was supported by State Key Projects Specialized on Infectious Diseases (No. 2017ZX10201201-006), Key research projects for precision medicine (No. 2017YFC0908103). Innovation Fund for Medical Sciences of Chinese Academy of Medical Sciences (CIFMS, 2019-I2M-2-004, Cohort study of the esophageal cancer and liver cancer; and CIFMS, 2016-I2M-1-007, 2019-I2M-1-003), and National Natural Science Foundation Fund (81972628). The study sponsors were not involved in this research design, data collection, analysis, interpretation, or manuscript submission.

Data Availability: The authors confirm that, for approved reasons, some access restrictions apply to the data underlying the findings. Data are available for researchers who meet the criteria for access to confidential data from the Ethics Committee, National Cancer Center /Cancer Hospital, Chinese Academy of Medical Sciences. The administrator at the Office of Science and Research, National Cancer Center/National Clinical Research Center for Cancer/Cancer Hospital, Chinese Academy of Medical Sciences can be contacted to data request by email: keyanchu@cicams.ac.cn and quchf@cicams.ac.cn.

Code availability: Not applicable.

\section{Compliance with ethical standards}

Conflicts of interest: Yuting Wang, Minjie Wang, He Li, Kun Chen, Hongmei Zeng, Xinyu Bi, Zheng Zhu, Yuchen Jiao, Yong Wang, Jian Zhu, Hui Zhao, Xiang Liu, Chunyun Dai, Chunsun Fan, Can Zhao, Deyin Guo, Hong Zhao, Sizhen Wang, Jianguo Zhou, Dongmei Wang, Zhiyuan Wu, Xinming Zhao, Wei Cui, Xuehong Zhang, Jianqiang Cai, Wanqing Chen and Chunfeng Qu declare that they have no conflict of interest.

Ethics approval: The study protocol (NCC201709011) was approved by the Ethical Committees of the National Cancer Center/ Cancer Hospital, Chinese Academy of Medical Sciences (NCC/CH-CAMS). All procedures performed in studies involving human participants were in accordance with the ethical standards of 1964 Helsinki declaration and its later amendments or comparable ethical standards.

Consent to participate: All participants provided written informed consent before any study procedures were provided.

Consent for publication: All authors of this manuscript have read and approved the final submitted version, and are aware that they are listed as an author on this paper.

Animal research: This article does not contain any studies with animals performed by any of the authors.

Clinical trials registration: The current study was based on the cohort of Community-based Cohort study on Population with high risk of Liver Cancer (Chinese Clinical Registry, ChiCTR-EOC-17012853).

Page 7/16 


\section{References}

1. Kulik L El-Serag HB. Epidemiology and Management of Hepatocellular Carcinoma. Gastroenterology 2019; 156:477-491 e471, Jan, 2019

2. Omata M, Cheng AL, Kokudo N, et al. Asia-Pacific clinical practice guidelines on the management of hepatocellular carcinoma: a 2017 update. Hepatol Int 2017; 11:317-370, Jul, 2017

3. Zhao LH, Liu X, Yan HX, et al. Genomic and oncogenic preference of HBV integration in hepatocellular carcinoma. Nat Commun 2016; 7:12992, Oct 5, 2016

4. Ji M, Liu Z, Chang ET, et al. Mass screening for liver cancer: results from a demonstration screening project in Zhongshan City, China. Sci Rep 2018; 8:12787, Aug 24, 2018

5. Thomas DL. Global Elimination of Chronic Hepatitis. N Engl J Med 2019; 380:2041-2050, May 23, 2019

6. Kansagara D, Papak J, Pasha AS, et al. Screening for hepatocellular carcinoma in chronic liver disease: a systematic review. Ann Intern Med 2014; 161:261-269, Aug 19, 2014

7. Fattovich G, Stroffolini T, Zagni I, et al. Hepatocellular carcinoma in cirrhosis: Incidence and risk factors. Gastroenterology 2004; 127:S35-S50, 2004/11/01/, 2004

8. Chen CJ, Yang HI, Su J, et al. Risk of hepatocellular carcinoma across a biological gradient of serum hepatitis B virus DNA level. Jama $2006 ; 295: 65-73,2006$

9. Yang HI, Sherman M, Su J, et al. Nomograms for risk of hepatocellular carcinoma in patients with chronic hepatitis B virus infection. J Clin Oncol 2010; 28:2437-2444, May 10, 2010

10. Wong VW-S, Chan SL, Mo F, et al. Clinical Scoring System to Predict Hepatocellular Carcinoma in Chronic Hepatitis B Carriers. Journal of Clinical Oncology 2010; 28:1660-1665, 2010/04/01, 2010

11. Yuen M-F, Tanaka Y, Fong DY-T, et al. Independent risk factors and predictive score for the development of hepatocellular carcinoma in chronic hepatitis B. Journal of hepatology 2009; 50:80-88, 2009/01/01/, 2009

12. Fan C, Li M, Gan Y, et al. A simple AGED score for risk classification of primary liver cancer: development and validation with long-term prospective HBsAg-positive cohorts in Qidong, China. Gut 2019; 68:948-949, May, 2019

13. Papatheodoridis G, Dalekos G, Sypsa V, et al. PAGE-B predicts the risk of developing hepatocellular carcinoma in Caucasians with chronic hepatitis B on 5-year antiviral therapy. J Hepatol 2016; 64:800-806, Apr, 2016

14. Kim JH, Kim YD, Lee M, et al. Modified PAGE-B score predicts the risk of hepatocellular carcinoma in Asians with chronic hepatitis B on antiviral therapy. J Hepatol 2018; 69:1066-1073, Nov, 2018

15. Voulgaris T, Papatheodoridi M, Lampertico P, et al. Clinical utility of hepatocellular carcinoma risk scores in chronic hepatitis B. Liver Int 2020; 40:484-495, Mar, 2020

16. Chen W, Sun K, Zheng R, et al. Cancer incidence and mortality in China, 2014. Chin J Cancer Res 2018; 30:1-12, 2018

17. Chen JG, Parkin DM, Chen QG, et al. Screening for liver cancer: results of a randomised controlled trial in Qidong, China. J Med Screen 2003; 10:204-209, 2003

18. Sato T, Kondo F, Ebara M, et al. Natural history of large regenerative nodules and dysplastic nodules in liver cirrhosis: 28-year follow-up study. Hepatol Int 2015; 9:330-336, Apr, 2015

19. Wang SH, Yeh SH, Lin WH, et al. Estrogen receptor alpha represses transcription of HBV genes via interaction with hepatocyte nuclear factor 4alpha. Gastroenterology 2012; 142:989-998 e984, Apr, 2012

20. Li CL, Li CY, Lin YY, et al. Androgen Receptor Enhances Hepatic Telomerase Reverse Transcriptase Gene Transcription After Hepatitis B Virus Integration or Point Mutation in Promoter Region. Hepatology 2019; 69:498-512, Feb, 2019

21. Saxena S Wong ET. Heterogeneity of common hematologic parameters among racial, ethnic, and gender subgroups. Arch Pathol Lab Med 1990; 114:715-719, 1990

22. Wu WC, Wu CY, Wang YJ, et al. Updated thresholds for serum alanine aminotransferase level in a large-scale population study composed of 34346 subjects. Aliment Pharmacol Ther 2012; 36:560-568, Sep, 2012

23. Qu C, Wang Y, Wang P, et al. Detection of early-stage hepatocellular carcinoma in asymptomatic HBsAg-seropositive individuals by liquid biopsy. Proc Natl Acad Sci U S A 2019; 116:6308-6312, Mar 26, 2019

24. Sharma SA, Kowgier M, Hansen BE, et al. Toronto HCC risk index: A validated scoring system to predict 10-year risk of HCC in patients with cirrhosis. J Hepatol 2017; 24:32248-32241, 2017

25. Johnson PJ, Pirrie SJ, Cox TF, et al. The detection of hepatocellular carcinoma using a prospectively developed and validated model based on serological biomarkers. Cancer Epidemiol Biomarkers Prev 2014; 23:144-153, 2014 
26. Lok AS, Sterling RK, Everhart JE, et al. Des-gamma-carboxy prothrombin and alpha-fetoprotein as biomarkers for the early detection of hepatocellular carcinoma. Gastroenterology 2010; 138:493-502, Feb, 2010

27. Chen H, Zhang Y, Li S, et al. Direct comparison of five serum biomarkers in early diagnosis of hepatocellular carcinoma. Cancer Manag Res 2018; 10:1947-1958, 2018

28. Tayob N, Christie I, Richardson P, et al. Validation of the Hepatocellular Carcinoma Early Detection Screening (HES) Algorithm in a Cohort of Veterans With Cirrhosis. Clinical Gastroenterology and Hepatology 2019; 17:1886-1893.e1885, 2019/08/01/, 2019

29. Fan C, Zhu J, Wang Y, et al. Analysis of risk factors for primary liver cancer in rural China and high risk population identification: a cohort study in Qidong, China. Chin J Evi-Based Med 2018; 18:428-433, 2018

30. Van Hemelrijck M, Jassem W, Walldius G, et al. Gamma-glutamyltransferase and risk of cancer in a cohort of 545,460 persons - the Swedish AMORIS study. Eur J Cancer 2011; 47:2033-2041, Sep, 2011

\section{Tables}


Table 1 Baseline characteristics of training and validation cohorts, number (\%)

\begin{tabular}{|c|c|c|c|c|}
\hline \multirow[t]{2}{*}{ Factors } & & Training & Validation & \multirow[t]{2}{*}{$P$-value } \\
\hline & & $(\mathrm{N}=3$ 629) & $(\mathrm{N}=2524)$ & \\
\hline HCC cases & & $212(5.84)$ & $90(3.57)$ & $<0.001$ \\
\hline \multicolumn{5}{|l|}{ Age, years } \\
\hline Median & & 51 & 54 & \\
\hline \multicolumn{5}{|l|}{ Range } \\
\hline & $<44$ & $810(22.32)$ & 379 (15.02) & \multirow[t]{4}{*}{$<0.001$} \\
\hline & $45-54$ & $1575(43.40)$ & $890(35.26)$ & \\
\hline & $55-64$ & $906(24.97)$ & 873 (34.59) & \\
\hline & $\geq 65$ & 338 (9.31) & $382(15.13)$ & \\
\hline
\end{tabular}

AFP, $\mathrm{ng} / \mathrm{mL}$

\begin{tabular}{llll}
$<7$ & $3206(88.34)$ & $2309(91.48)$ & $<0.001$ \\
$7-19.9$ & $257(7.08)$ & $145(5.74)$ \\
\hline $20-199.9$ & $113(3.11)$ & $43(1.70)$ \\
$\geq 200$ & $53(1.46)$ & $27(1.07)$
\end{tabular}

$\mathrm{DCP}, \mathrm{mAU} / \mathrm{mL}$

$\begin{array}{llll}<40 & 3510(96.72) & 2455(97.26) & 0.189 \\ 40-139.9 & 48(1.32) & 35(1.39) & \\ \geq 140 & 71(1.96) & 34(1.35)\end{array}$

GGT, U/L

$\begin{array}{llll}<15 & 1022(28.16) & 474(18.78) & <0.001 \\ 15-44 & 1954(53.84) & 1572(62.28) \\ 45-79 & 400(11.02) & 294(11.65) \\ \geq 80 & 253(6.97) & 184(7.29)\end{array}$

PLT, $10^{9}$

$\begin{array}{llll}<100 & 409(11.27) & 212(8.40) & 0.001 \\ 100-299 & 3094(85.26) & 2227(88.23) \\ \geq 300 & 126(3.47) & 85(3.37)\end{array}$

WBC, $10^{6}$

$\begin{array}{llll}<4 & 395(10.88) & 194(7.69) & <0.001 \\ 4-9.9 & 3145(86.66) & 2251(89.18) & \\ \geq 10 & 89(2.45) & 79(3.13)\end{array}$

ALB, $g / L$

$\begin{array}{llll}<30 & 11(0.30) & 5(0.20) & <0.001 \\ 30-37.9 & 146(4.02) & 65(2.58) & \\ 38-44.9 & 1498(41.28) & 609(24.13) \\ \geq 45 & 1974(54.40) & 1845(73.10)\end{array}$




\begin{tabular}{|c|c|c|c|}
\hline Yes & $587(16.18)$ & $266(10.54)$ & $<0.001$ \\
\hline \multicolumn{4}{|l|}{ BMI $\left(k g / m^{2}\right)$} \\
\hline$<18.5$ & $21(0.58)$ & $51(2.02)$ & \multirow[t]{4}{*}{$<0.001$} \\
\hline $18.5-24$ & $1361(37.50)$ & 1004 (39.78) & \\
\hline $24-27$ & $1264(34.83)$ & $844(33.44)$ & \\
\hline$>27$ & 983 (27.09) & $625(24.76)$ & \\
\hline Smoking (ever) & $1954(53.84)$ & $1397(55.35)$ & 0.244 \\
\hline Alcohol drinking (ever) & $1230(33.89)$ & $1124(44.53)$ & $<0.001$ \\
\hline Self-reported antiviral therapy received a & $982(27.06)$ & $583(23.10)$ & $<0.001$ \\
\hline Self-reported diabetes & $228(6.28)$ & $171(6.77)$ & 0.441 \\
\hline HCC family history & $363(10.00)$ & $431(17.08)$ & $<0.001$ \\
\hline \multicolumn{4}{|c|}{$\begin{array}{l}\text { HCC hepatocellular carcinoma; } A F P \text { a-fetoprotein; } D C P \text { des- } \gamma \text {-carboxy-prothrombin; } G G T \text { y-glutamyl-transpeptidase; } P L T \\
\text { platelets; } W B C \text { white blood cells; } A L B \text { albumin; US B-ultrasonography; } B M / \text { body mass index. }\end{array}$} \\
\hline
\end{tabular}


Table 2 The odds ratio (OR), $\beta$ coefficient of multivariate logistic regression analysis and point-based scoring system of Male-ABCD

$\begin{array}{llll}\text { Factors } & \beta \text { coefficient } & P \text {-value } & \begin{array}{l}\text { Points } \\ \text { assigned }\end{array}{ }^{c}\end{array}$

Age, years

\begin{tabular}{lllll}
$35-44$ & Reference & \multicolumn{2}{c}{0} \\
$45-59$ & 3.03 & 1.11 & 0.003 & 1.3 \\
$\geq 60$ & 6.70 & 1.90 & $<0.001$ & 2.2
\end{tabular}

AFP, $n g / m L$

\begin{tabular}{lllll}
$<7$ & Reference & & \multicolumn{2}{c}{0} \\
\hline $7-19.9$ & 2.52 & 0.92 & 0.001 & 1.1 \\
\hline $20-399.9$ & 3.98 & 1.38 & $<0.001$ & 1.6 \\
$\geq 400$ & 99.97 & 4.60 & $<0.001$ & 5.4
\end{tabular}

$\mathrm{DCP}, \mathrm{mAU} / \mathrm{mL}$

\begin{tabular}{lllll}
$<20$ & Reference & & \multicolumn{2}{c}{0} \\
$20-39.9$ & 3.98 & 1.38 & $<0.001$ & 1.6 \\
\hline $40-49.9$ & 20.15 & 3.00 & $<0.001$ & 3.5 \\
$50-119.9$ & 91.07 & 4.51 & $<0.001$ & 5.3 \\
$\geq 120$ & 224.66 & 5.41 & $<0.001$ & 6.3
\end{tabular}

GGT, U/L

\begin{tabular}{lllll}
$<20$ & Reference & & \multicolumn{2}{c}{0} \\
$20-44$ & 2.52 & 0.92 & $<0.001$ & 1.1 \\
$45-79$ & 3.18 & 1.16 & $<0.001$ & 1.4 \\
$\geq 80$ & 2.57 & 0.94 & 0.009 & 1.1
\end{tabular}

PLT, $10^{9}$

\begin{tabular}{lllll}
$\geq 150$ & Reference & \multicolumn{3}{c}{0} \\
$80-149$ & 2.35 & 0.85 & $<0.001$ & 1 \\
$<80$ & 5.18 & 1.64 & $<0.001$ & 1.9
\end{tabular}

WBC, $10^{6}$

\begin{tabular}{|cllll}
\hline$\geq 4$ & Reference & \multicolumn{2}{c}{0} \\
\hline$<4$ & 2.56 & 0.94 & $<0.001$ & 1.1 \\
\hline Intercept & - & -6.64 & $<0.001$ & -
\end{tabular}

$O R$ odds ratio; $C /$ confidential interval; $A F P$ a-fetoprotein; $D C P$ des-y-carboxy-prothrombin; GGT $\gamma$-glutamyl transpeptidase; PLT platelets; $W B C$ white blood cells.

a The Chi-square of Hosmer-Lemeshow Test is $3.77(P=0.88)$

b 1000 bootstrap samples were generated by using resampling with replacement

${ }^{\mathrm{c}}$ The points were assigned by dividing the coefficient of each variable by the smallest coefficient in the model (0.85) and rounding to the nearest 0.1 , for example, the coefficient for age $45-59$ years was 1.11 , and the smallest coefficient in the model was 0.85 (PLT, 80 to 149$)$, so age $45-59$ years were assigned 1.3 points $(1.11 / 0.85)$ 


\begin{tabular}{|c|c|c|c|c|c|c|c|c|c|c|}
\hline \multirow{3}{*}{$\begin{array}{l}\text { Cutoff } \\
\text { of risk } \\
\text { score }\end{array}$} & \multicolumn{5}{|c|}{ Training cohort } & \multicolumn{5}{|c|}{ Validation cohort } \\
\hline & \multirow[t]{2}{*}{ Sensitivity } & \multicolumn{2}{|l|}{ at-high-risk } & \multicolumn{2}{|l|}{ at-low-risk } & \multirow[t]{2}{*}{ Sensitivity } & \multicolumn{2}{|l|}{ at-high-risk } & \multicolumn{2}{|l|}{ at-low-risk } \\
\hline & & Proportion & PPV & Proportion & NPV & & Proportion & PPV & Proportion & NPV \\
\hline 0.5 & 100.00 & 94.46 & 6.18 & 5.54 & 100.00 & 100.00 & 96.87 & 3.68 & 3.13 & 100.00 \\
\hline 1.0 & 100.00 & 94.41 & 6.19 & 5.59 & 100.00 & 100.00 & 96.87 & 3.68 & 3.13 & 100.00 \\
\hline 1.5 & 100.00 & 73.90 & 7.90 & 26.10 & 100.00 & 100.00 & 85.06 & 4.19 & 14.94 & 100.00 \\
\hline 2.0 & 99.53 & 71.07 & 8.18 & 28.93 & 99.90 & 98.89 & 83.40 & 4.23 & 16.60 & 99.76 \\
\hline 2.5 & 99.06 & 53.49 & 10.82 & 46.51 & 99.88 & 97.78 & 66.32 & 5.26 & 33.68 & 99.76 \\
\hline 3.0 & 97.17 & 44.53 & 12.75 & 55.47 & 99.70 & 95.56 & 55.74 & 6.11 & 44.26 & 99.64 \\
\hline 3.5 & 94.81 & 35.93 & 15.41 & 64.07 & 99.53 & 92.22 & 43.94 & 7.48 & 56.06 & 99.51 \\
\hline 4.0 & 88.21 & 29.15 & 17.67 & 70.85 & 99.03 & 87.78 & 32.37 & 9.67 & 67.63 & 99.36 \\
\hline 4.5 & 81.60 & 20.86 & 22.85 & 79.14 & 98.64 & 82.22 & 23.73 & 12.35 & 76.27 & 99.17 \\
\hline 5.0 & 74.06 & 15.40 & 28.09 & 84.60 & 98.21 & 73.33 & 16.32 & 16.02 & 83.68 & 98.86 \\
\hline 5.5 & 68.40 & 11.35 & 35.19 & 88.65 & 97.92 & 66.67 & 12.00 & 19.80 & 88.00 & 98.65 \\
\hline 6.0 & 58.96 & 7.85 & 43.86 & 92.15 & 97.40 & 58.89 & 7.49 & 28.04 & 92.51 & 98.42 \\
\hline 6.5 & 53.30 & 5.81 & 53.55 & 94.19 & 97.10 & 53.33 & 5.78 & 32.88 & 94.22 & 98.23 \\
\hline 7.0 & 48.58 & 4.02 & 70.55 & 95.98 & 96.87 & 48.89 & 4.00 & 43.56 & 96.00 & 98.10 \\
\hline 8.0 & 47.17 & 3.17 & 86.96 & 96.83 & 96.81 & 40.00 & 2.58 & 55.38 & 97.42 & 97.80 \\
\hline 9.0 & 42.45 & 2.70 & 91.84 & 97.30 & 96.54 & 35.56 & 1.94 & 65.31 & 98.06 & 97.66 \\
\hline 10.0 & 35.38 & 2.20 & 93.75 & 97.80 & 96.14 & 28.89 & 1.43 & 72.22 & 98.57 & 97.43 \\
\hline 11.0 & 27.83 & 1.71 & 95.16 & 98.29 & 95.71 & 25.56 & 1.11 & 82.14 & 98.89 & 97.32 \\
\hline 12.0 & 20.28 & 1.21 & 97.73 & 98.79 & 95.29 & 23.33 & 0.99 & 84.00 & 99.01 & 97.24 \\
\hline 13.0 & 15.57 & 0.94 & 97.06 & 99.06 & 95.02 & 17.78 & 0.71 & 88.89 & 99.29 & 97.05 \\
\hline 14.0 & 12.74 & 0.74 & 100.00 & 99.26 & 94.86 & 13.33 & 0.52 & 92.31 & 99.48 & 96.89 \\
\hline 15.0 & 7.55 & 0.44 & 100.00 & 99.56 & 94.58 & 10.00 & 0.36 & 100.00 & 99.64 & 96.78 \\
\hline 16.0 & 4.72 & 0.28 & 100.00 & 99.72 & 94.42 & 5.56 & 0.20 & 100.00 & 99.80 & 96.63 \\
\hline 17.0 & 1.42 & 0.08 & 100.00 & 99.92 & 94.24 & 1.11 & 0.04 & 100.00 & 99.96 & 96.47 \\
\hline 17.5 & 0.00 & 0.00 & Undefined & 100.00 & 94.16 & 0.00 & 0.00 & Undefined & 100.00 & 96.43 \\
\hline
\end{tabular}


Table 4 Performance of Male-ABCD and some established models for HCC prediction and stratification among 6153 HBsAg-positive males

Models of HCC prediction

Cutoff a $\quad$ Sensitivity (\%)

Predicted proportion (\%) at different HCC risk

PPV (\%) NPV (\%)

High Low

Male-ABCD (18.3 points in total)

\begin{tabular}{llllll}
1.5 points & 100.00 & 78.48 & 21.52 & 6.25 & 100.00 \\
\hline 2.5 points & 98.68 & 58.75 & 41.25 & 8.24 & 99.84 \\
\hline 4.5 points & 81.79 & 22.04 & 77.96 & 18.22 & 98.85
\end{tabular}

AGED $^{b}$ (12 points in total)

\begin{tabular}{llllll}
\hline 4 points & 99.06 & 81.45 (intermediate) & 6.37 & 6.18 & 99.13 \\
\hline 9 points & 12.18 (high) & &
\end{tabular}

REACH-B ${ }^{b}$ (17 points in total)

\begin{tabular}{llllll}
5 points & 100.00 & 76.22 (intermediate) & 4.27 & 6.10 & 100.00 \\
\hline 11 points & 19.51 (high) & & &
\end{tabular}

PAGE-B (25 points in total)

$\begin{array}{llllll}10 \text { points } & 100.00 & 52.41 \text { (intermediate) } & 3.17 & 5.07 & 100.00 \\ 18 \text { points } & 44.42 \text { (high) } & & \end{array}$

mPAGE-B (21 points in total)

$\begin{array}{llllll}8 \text { points } & 99.01 & 54.61 \text { (intermediate) } & 9.25 & 5.35 & 99.47 \\ 13 \text { points } & 36.14 \text { (high) } & & \end{array}$

THRI (366 points in total)

$\begin{array}{lllll}120 \text { points } & 100.00 & 32.91 \text { (intermediate) } & 0.00 & 4.91 \\ 240 \text { points } & 67.09 \text { (high) } & \end{array}$

GALAD

$\begin{array}{llccc}Z^{c}=-1.36 & 90.73 & - & 8.39 & 99.03 \\ Z=-0.63 & 80.79 & - & 13.27 & 98.66 \\ Z=0.88 & 52.98 & - & 42.44 & 97.54\end{array}$

$H B s A g$ hepatitis B surface antigen; HCC hepatocellular carcinoma; AUROC area under the receiver operating characteristics; $P P V$ positive predictive value; $N P V$ negative predictive value

AGED: Age, Gender, HBeAg, HBV-DNA [12];

REACH-B: Age, Gender, ALT, HBeAg, HBV-DNA [9];

PAGE-B: Age, Gender, PLT [13];

mPAGE-B: Age, Gender, PLT, ALB [14];

THRI: Age, Gender, Etiology, PLT [24];

GALAD: Age, Gender, AFP, AFP-L3, DCP [25]

a The cutoff values for AGED, REACH-B, PAGE-B, mPAGE-B, THRI and GALAD were selected as recommended from the original studies

b HBV DNA was only detected in training cohort. The performance of AGED and REACH-B was calculated among training cohort of 3629 HBsAg-positive males

${ }^{c} Z=-10.08+0.09 *$ age $+1.67 *$ sex $+2.34 * \log (A F P)+0.04 * A F P-L 3+1.33 * \log (D C P)$ 
Figures

Baseline screening in 2017
HBsAg-positive males at age 35-69 years from 6 community-based $\mathrm{HCC}$-screening centers

\section{I}

I

I Annually screening and

I follow-up for 24 months

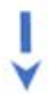

$$
(\mathrm{N}=6,876)
$$

Exclusion

History of $\mathrm{HCC}(\mathrm{N}=87)$

Other cancers $(\mathrm{N}=70)$

Loss of follow-up ( $\mathrm{N}=496)$

Death $(\mathrm{N}=70)$
Last follow-up

in 2019

\section{6,153 participants were eligible}

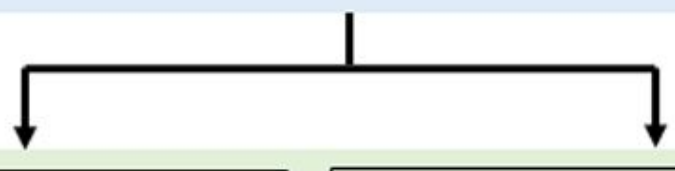

\begin{tabular}{|c|}
\hline Training cohort \\
$(3$ centers, $\mathrm{N}=3,629$, \\
$212 \mathrm{HCC})$
\end{tabular}

Validation cohort

( 3 centers, $\mathrm{N}=2,524$, $90 \mathrm{HCC}$ )

Male-ABCD algorithm development and validation (Age, AFP, DCP, GGT, PLT, WBC)

Figure 1

Flowchart of the study population from multicenter population-based liver cancer screening. HCC hepatocellular carcinoma; AFP afetoprotein; DCP des-y-carboxy-prothrombin; GGT y-glutamyl-transpeptidase; PLT platelets; WBC white blood cells 

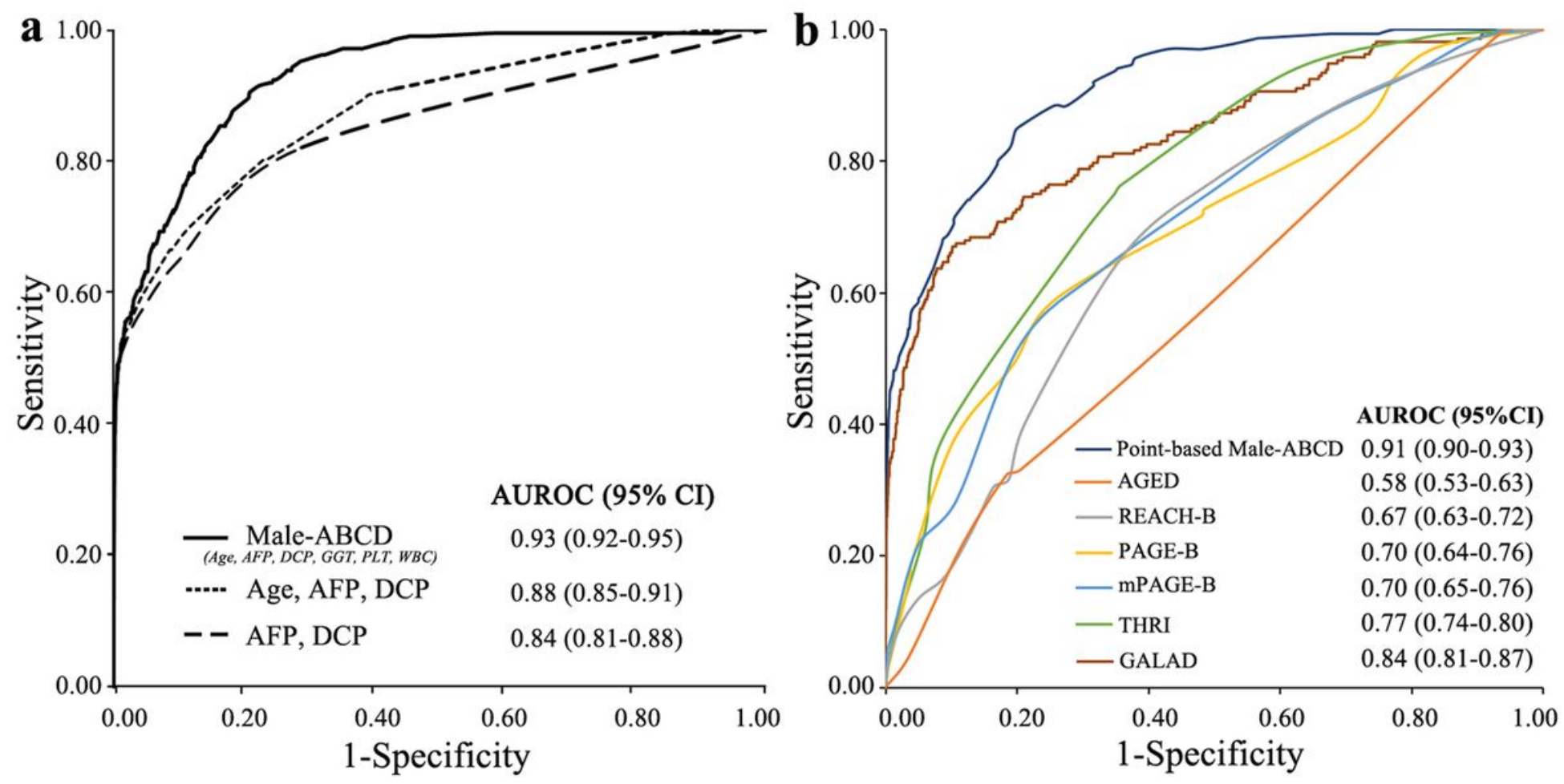

Figure 2

Receiver operator characteristic curves of Male-ABCD. a. Comparison of Male-ABCD with the combinations of age, AFP and DCP in training cohort. b. Comparison of Male-ABCD with some established models among $6153 \mathrm{HBsAg-positive} \mathrm{males.} \mathrm{AUROC} \mathrm{area} \mathrm{under} \mathrm{the} \mathrm{receiver}$ operating characteristics; AFP a-fetoprotein; DCP des-y-carboxy-prothrombin; GGT y-glutamyl-transpeptidase; PLT platelets; WBC white blood cells

\section{Supplementary Files}

This is a list of supplementary files associated with this preprint. Click to download.

- GraphicalAbstract.jpg

- Suptable0118.docx 